\title{
Perfluorohexane-loaded polymeric nanovesicles with oxygen supply for enhanced sonodynamic therapy
}

\footnotetext{
Qiang Zeng ${ }^{\mathrm{a}, 1}$, Lijuan Qiao ${ }^{\mathrm{a}, 1}$, Lili Cheng ${ }^{\mathrm{a}}$, Chao $\mathrm{Li}^{\mathrm{a}}$, Zhong Cao ${ }^{\mathrm{a}}$, Zhiyi Chen ${ }^{\mathrm{b}, \mathrm{c}}$, Yi Wang,c, Jie Liu, ${ }^{\mathrm{a}, *}$

a School of Biomedical Engineering, Sun Yat-sen University, Guangzhou, Guangdong, 510006, China

${ }^{b}$ Laboratory of Ultrasound Molecular Imaging, Department of Ultrasound Medicine, The Third Affiliated Hospital of Guangzhou Medical University, Guangzhou, PR China;

${ }^{c}$ Laboratory of Ultrasound Molecular Imaging, Department of Ultrasound Medicine, The Liwan Hospital of the Third Affiliated Hospital of Guangzhou Medical University, Guangzhou, PR China.
}

* Corresponding author. Jie Liu, Ph.D

E-mail address: 1iujie56@mail.sysu.edu.cn

${ }^{1}$ These authors contributed equally to this work. 


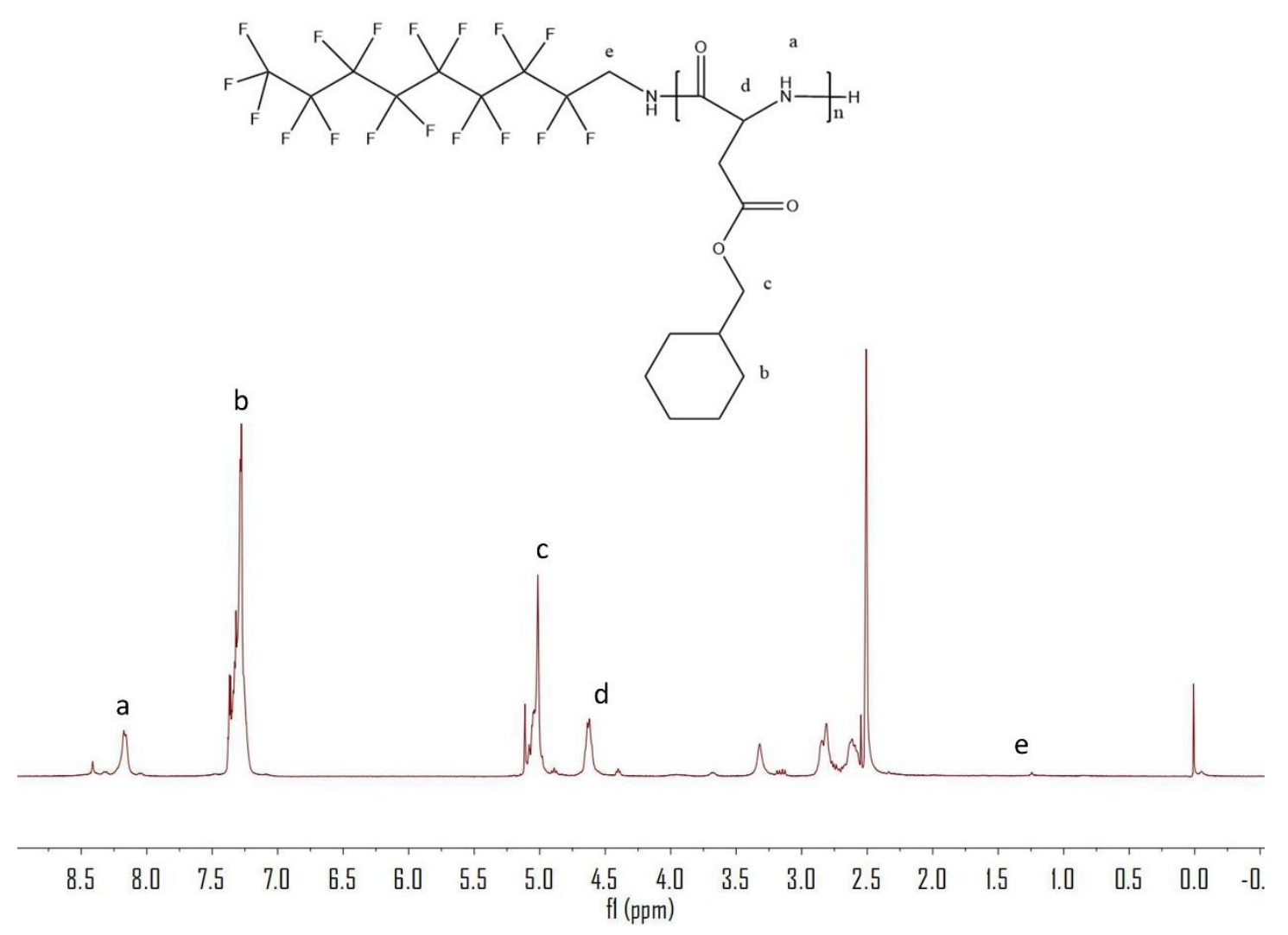

Figure S1. ${ }^{1} \mathrm{H}-\mathrm{NMR}$ Spectrum of $\mathrm{C}_{9} \mathrm{~F}_{17}$-PBLA. A: 8.20 (br.), B: 7.30 (br., 5H), C:5.07(br., 4H), D: 4.62(br.), E:1.24(s). (DMSO-d 6 ; ppm) 


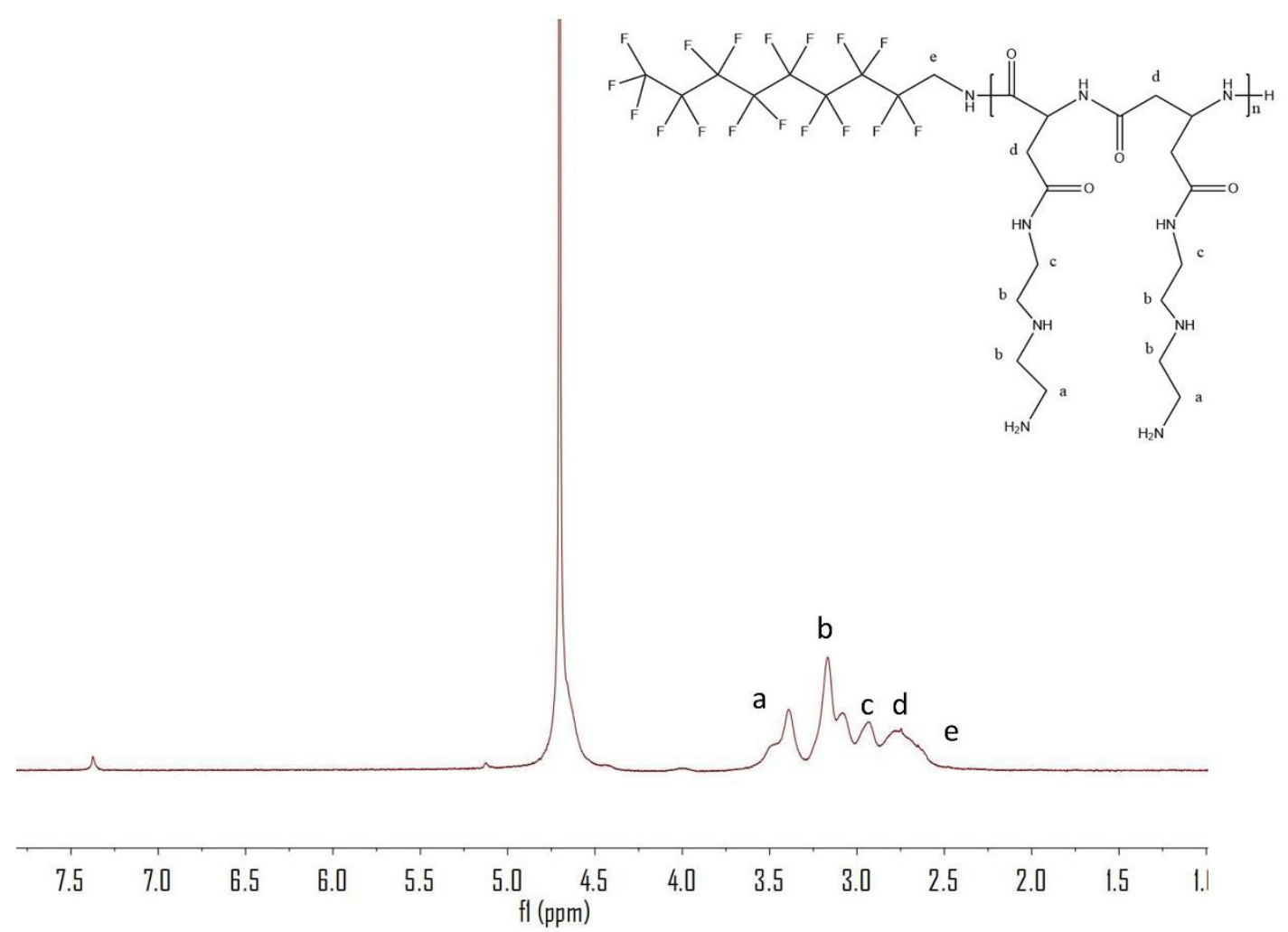

Figure S2. ${ }^{1} \mathrm{H}-\mathrm{NMR}$ Spectrum of $\mathrm{C}_{9} \mathrm{~F}_{17}-\mathrm{PAsp}(\mathrm{DET})$. A: 3.4 (br. , 4H), B: 3.09-3.17 (d, br., 8H), C: 3.10(br., 4H), D: 2.92(br., 2H), E: 2.75(s). ( $\left.\mathrm{D}_{2} \mathrm{O} ; \mathrm{ppm}\right)$ 


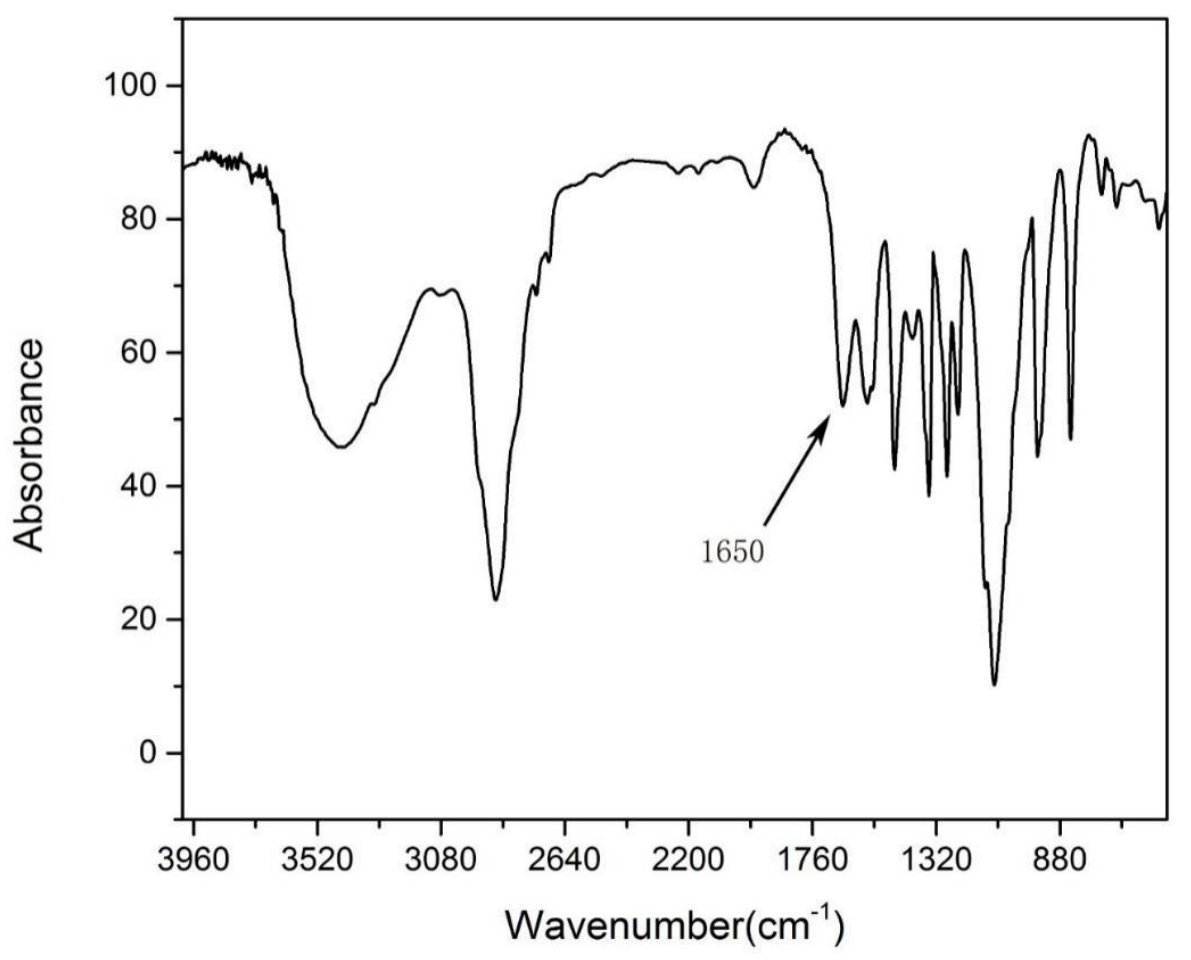

Figure S3. FT-IR Spectrum of mPEG-PpIX. 

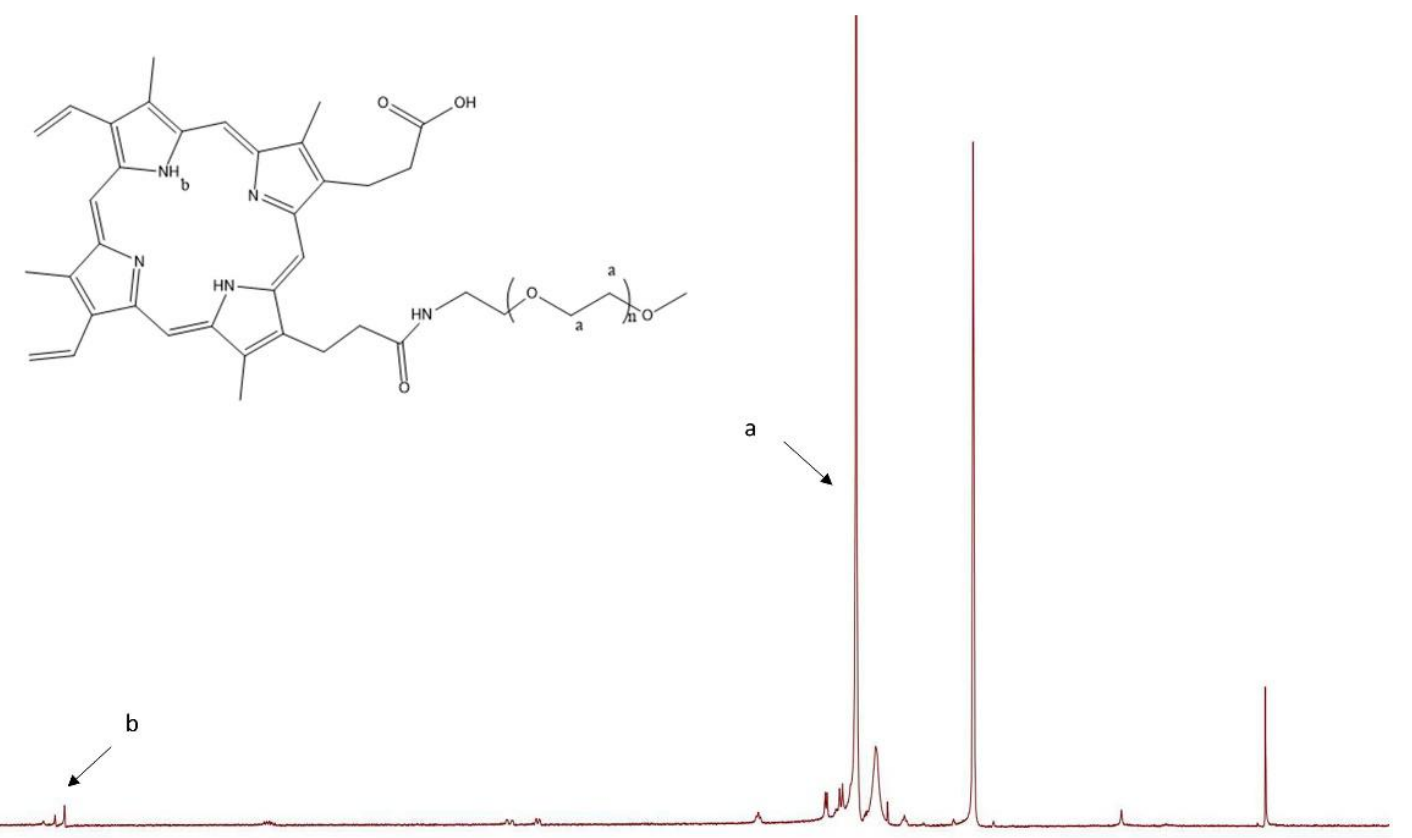

$\begin{array}{lllllllllllllllllllllllll}10.5 & 10.0 & 9.5 & 9.0 & 8.5 & 8.0 & 7.5 & 7.0 & 6.5 & 6.0 & 5.5 & 5.0 & 4.5 & 4.0 & 3.5 & 3.0 & 2.5 & 2.0 & 1.5 & 1.0 & 0.5 & 0.0 & -0.5 & -1.1\end{array}$

Figure S4. ${ }^{1} \mathrm{H}-\mathrm{NMR}$ Spectrum of mPEG-PpIX. A: 3.4 (br., 4H), B: 10.30 (br., 2H). $\left(\mathrm{DMSO}_{6} ; \mathrm{ppm}\right)$ 


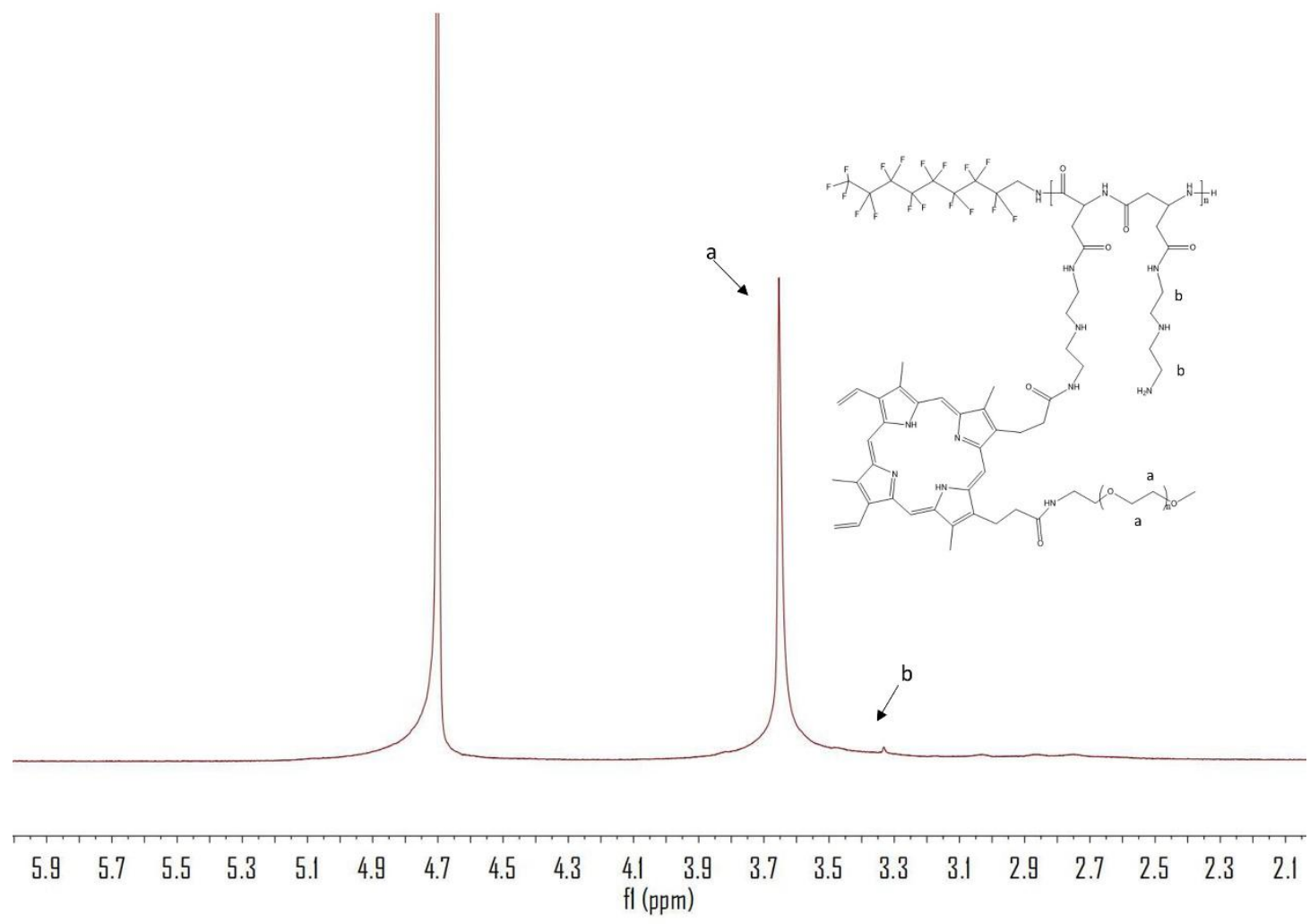

Figure S5. ${ }^{1} \mathrm{H}-\mathrm{NMR}$ Spectrum of PAsp(DET)-PpIX-PEG. A; 3.65 (br. 4H), B: 3.37 (br., $8 \mathrm{H}) .\left(\mathrm{D}_{2} \mathrm{O} ; \mathrm{ppm}\right)$ 


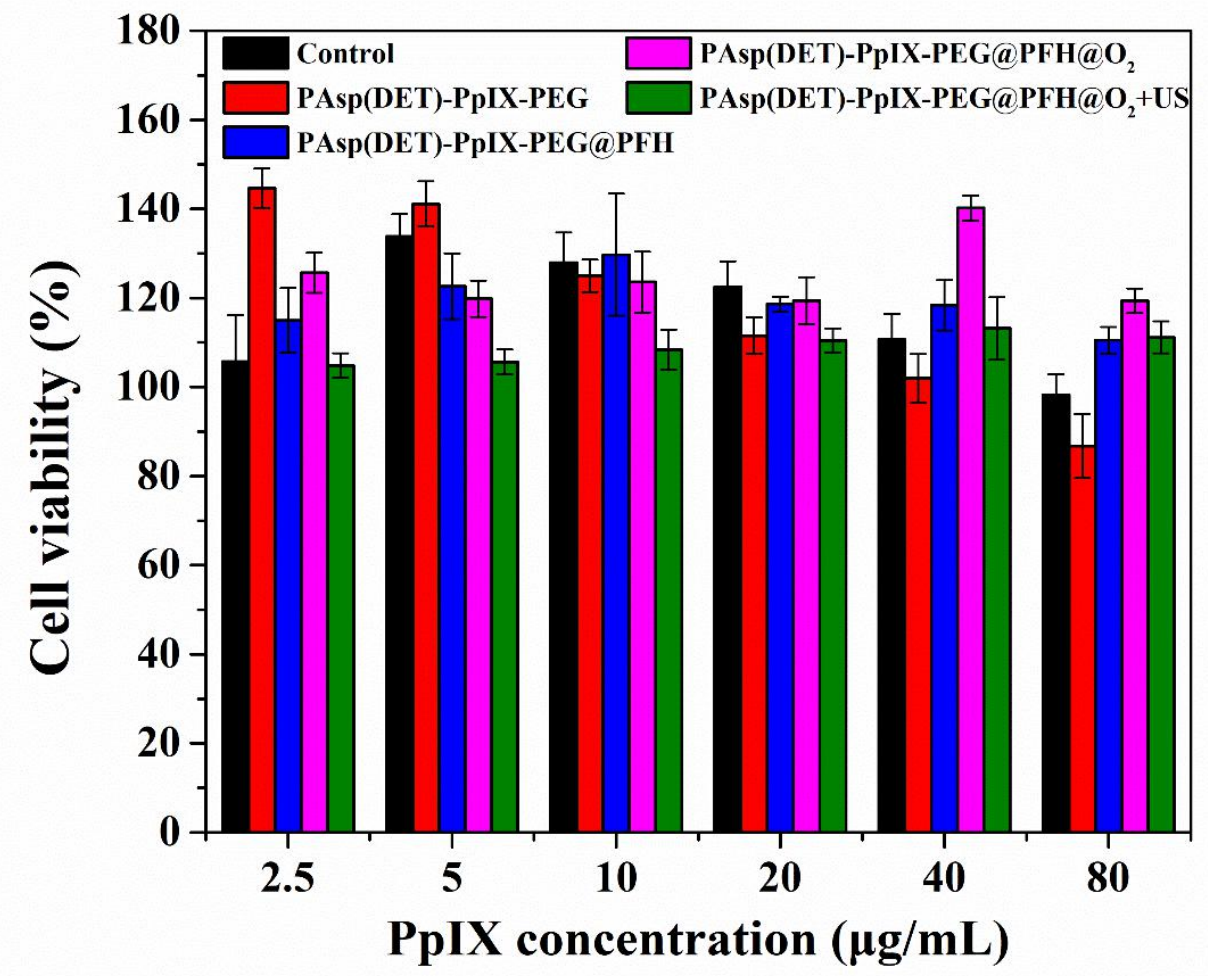

Figure S6. Cytotoxicity of $0.9 \% \mathrm{NaCl}$ (Control), PAsp(DET)-PpIX-PEG, PAsp(DET)-PpIX-PEG@PFH and PAsp(DET)-PpIX-PEG@PFH@O $\mathrm{O}_{2}$ after the incubation with $3 \mathrm{~T} 3$ cells for $48 \mathrm{H}$. Error bars represent standard deviation $(\mathrm{n}=5$ per group). 

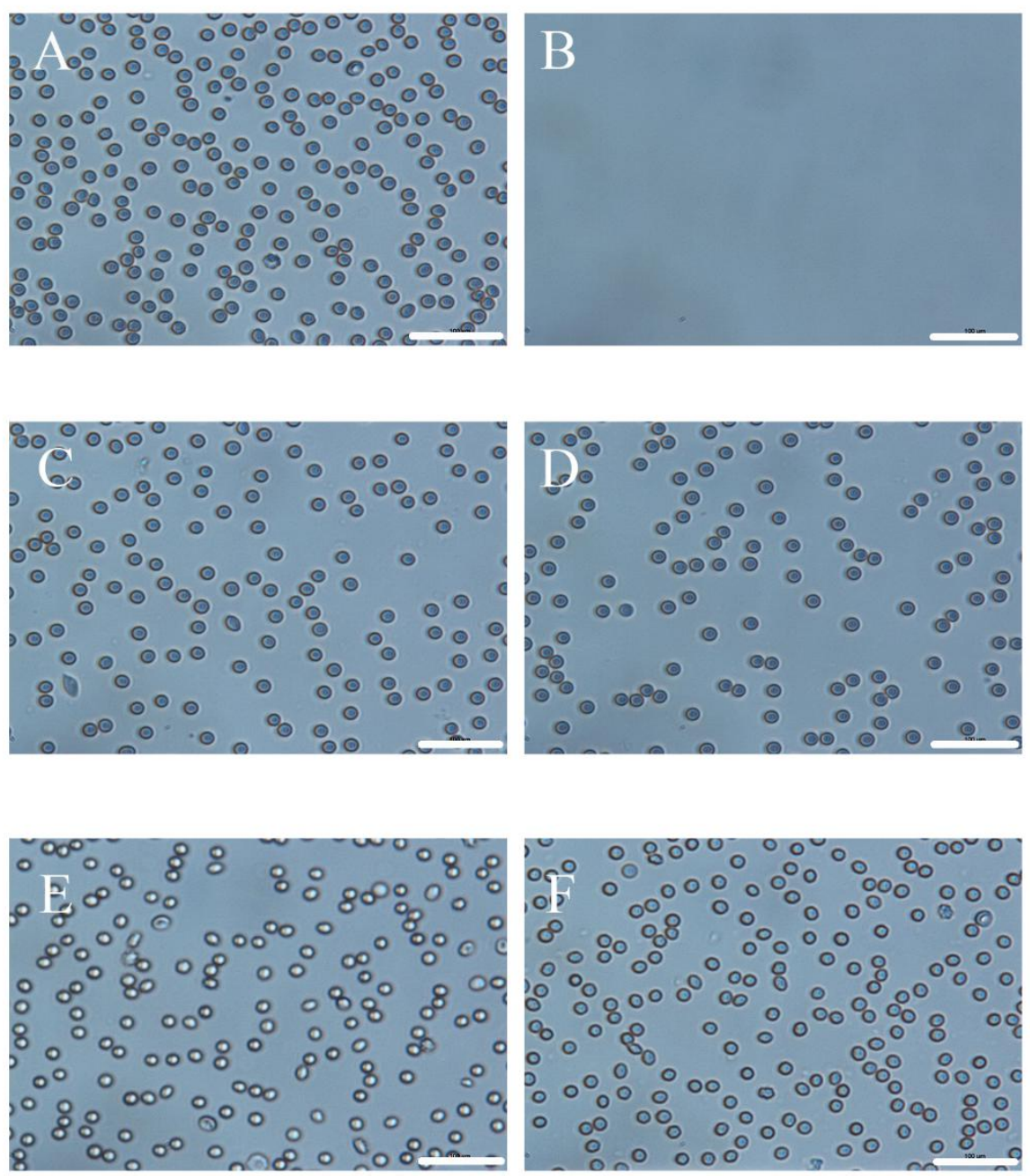

Figure S7. Erythrocyte images after incubated with different Nano vesicles for $2 \mathrm{~h}$.

(A)PBS, (B)Triton X-100, PAsp(DET)-PpIX-PEG and PAsp(DET)-PpIX-PEG@PFH at $40 \mu \mathrm{g} / \mathrm{mL}(\mathrm{C}, \mathrm{D})$ and $20 \mu \mathrm{g} / \mathrm{mL}$ (E, F). Scale bar: $100 \mu \mathrm{m}$. 


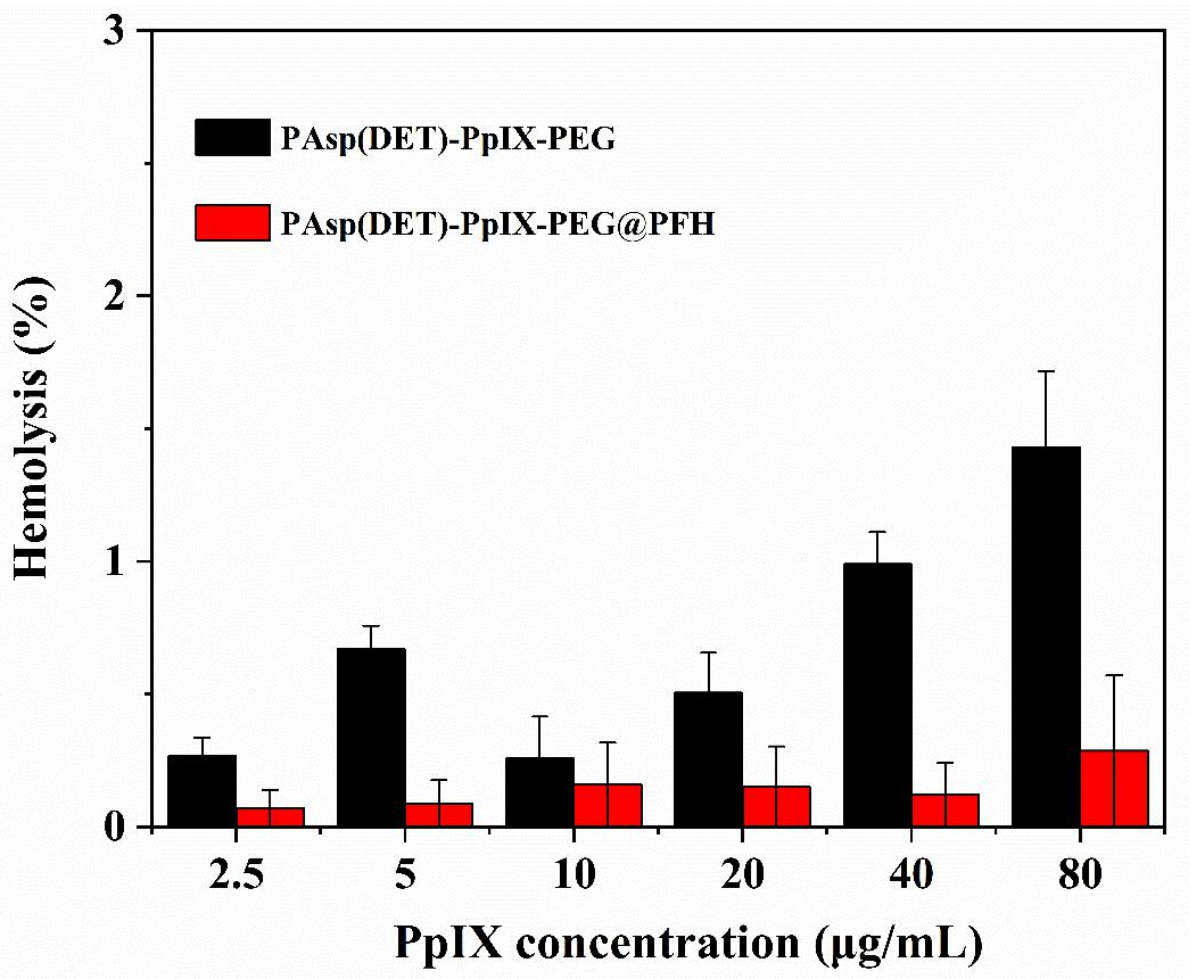

Figure S8. Hemolytic activity of PAsp(DET)-PpIX-PEG and PAsp(DET)-PpIX-PEG@PFH nanovesicles after incubated with erythrocytes for $2 \mathrm{~h}$. Error bars represent standard deviation ( $n=3$ per group). 


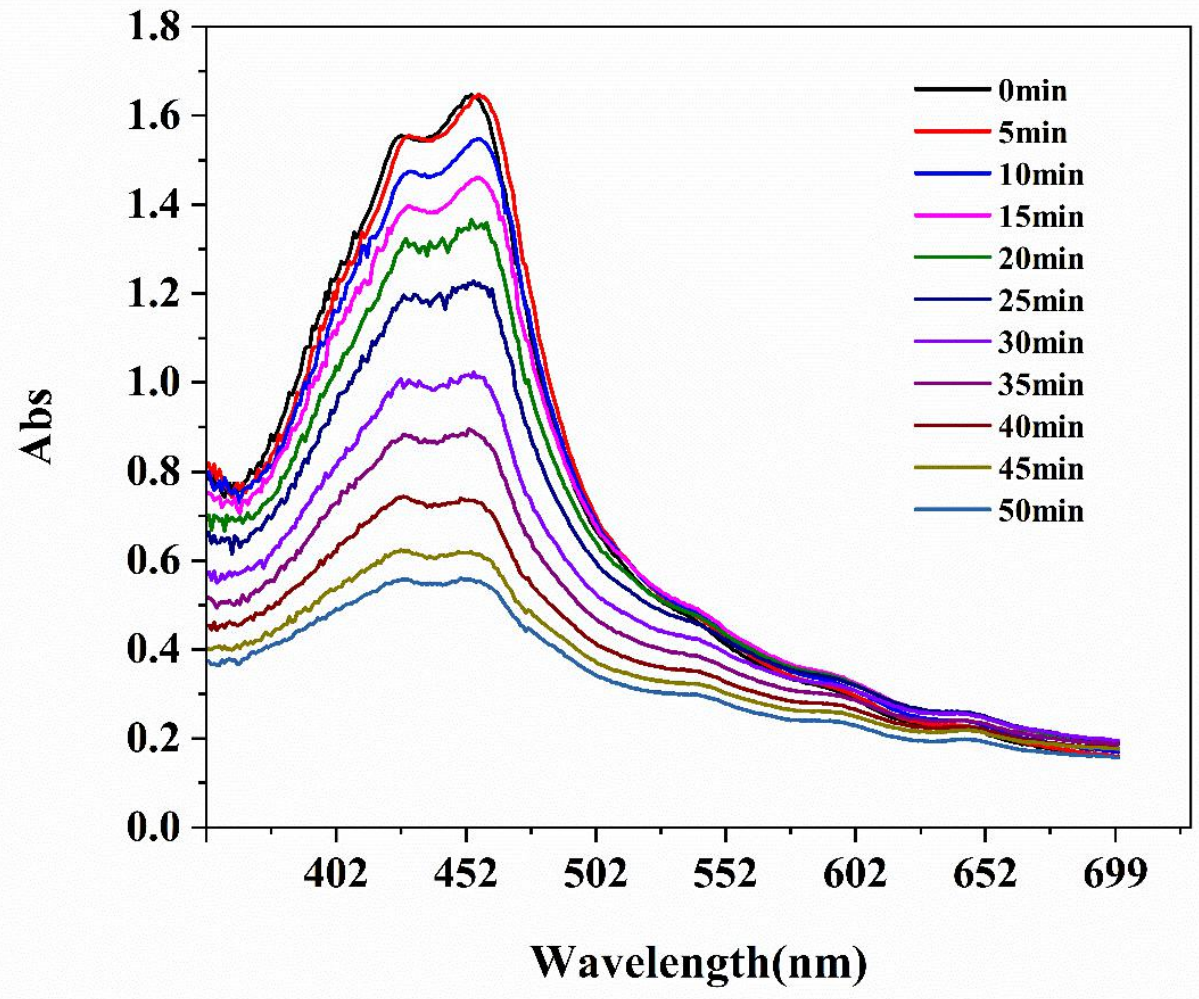

Figure S9. Time-dependent absorption spectra of the DPBF in the presence of PAsp(DET)-PpIX-PEG@PFH upon exposure to U.S irradiation for varied durations. 

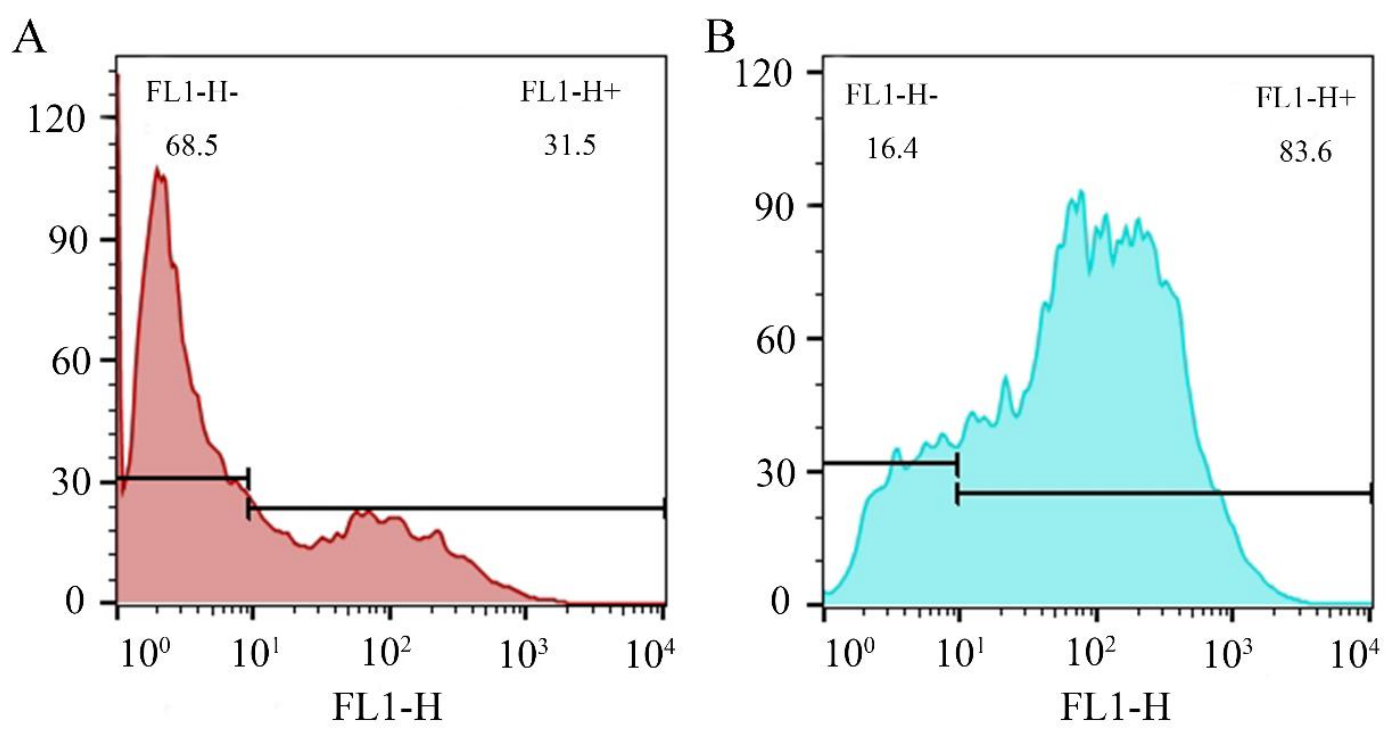

Figure S10. Flow cytometry for detection of intracellular ROS, before US irradiation (A) and after US irradiation (B). 